\title{
ARTICLE Selective dopamine D3 receptor antagonist YQA14 inhibits morphine-induced behavioral sensitization in wild type, but not in dopamine D3 receptor knockout mice
}

\author{
Yang $\mathrm{Lv}^{1,2}$, Rong-rong $\mathrm{Hu}^{3}$, Manyi Jing ${ }^{2}$, Tai-yun $\mathrm{Zhao}^{2}$, Ning $\mathrm{Wu}^{2}$, Rui Song ${ }^{2}$, Jin $\mathrm{Li}^{2}$ and Gang $\mathrm{Hu}^{1}$
}

\begin{abstract}
Increasing preclinical evidence demonstrates that dopamine D3 receptor (D3R) antagonists are a potential option for the treatment of drug addiction. The reinstatement of the addiction can be triggered by environmental stimuli that acquire motivational salience through repeated associations with the drug's effects. YQA14 is a novel D3R antagonist that has exhibited pharmacotherapeutic efficacy in reducing cocaine and amphetamine reward and relapse to drug seeking in mice. In this study we investigated the effects of YQA14 on morphine-induced context-specific locomotor sensitization in mice. We showed that repeated injection of YQA14 $(6.25-25 \mathrm{mg} / \mathrm{kg}$ every day ip) prior to morphine (10 mg/kg every day sc) not only inhibited the acquisition, but also significantly attenuated the expression of morphine-induced locomotor sensitization. Furthermore, in the expression phase, one single injection of YQA14 (6.25-25 mg/kg, ip) dose-dependently inhibited the expression of morphine-induced behavioral sensitization. Moreover, YQA14 inhibited the expression of morphine-induced behavioral sensitization in wild mice (WT), but not in D3R knockout (D3R ${ }^{-1-}$ )

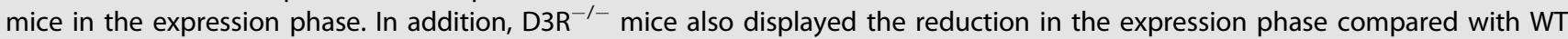
mice. In summary, this study demonstrates that blockade or knockout of the D3R inhibits morphine-induced behavior sensitization, suggesting that D3R plays an important role in the pathogenesis and etiology of morphine addiction, and it might be a potential target for clinical management of opioid addiction.
\end{abstract}

Keywords: morphine; dopamine D3 receptor; YQA14; behavioral sensitization; dopamine D3 receptor knockout mice

Acta Pharmacologica Sinica (2019) 40:583-588; https://doi.org/10.1038/s41401-018-0153-0

\section{INTRODUCTION}

Opioids, as potent analgesic drugs, are used for treating postoperative and cancer pain in clinical practice; however, the chronic use of morphine may lead to addiction, which induces harmful, long-lasting health consequences. Historically, opioid addiction has had greatly high rates in China. According to The Annual Report of Drug Abuse Monitoring in China in 2016, more than $45 \%$ of the addicts were opioid addicts [1]. Although several pharmacotherapies have been approved for the treatment of opioid addiction, such as methadone or buprenorphine $[2,3]$, they still have limits in the clinic $[2,4]$, including potential addiction and limited respiratory depression. Nevertheless, the development of novel, safer and more effective non-opioid medications for the treatment of prescription opioid abuse is a significant task.

Drug addiction is characterized by a high risk of relapse, which can persist for a lifetime. Usually, this is elicited by the environmental stimuli previously associated with the effects of abused drugs $[5,6]$. In animal studies, drug-induced locomotor sensitization is a well-established addiction model with good construct validity to investigate the process and mechanisms related to drug addiction [7]. Behavioral sensitization in the paired environment is commonly referred to as context-dependent sensitization. This sensitization is hypothesized to be due to aberrant learning processes in specific areas of the brain dopamine system due to chronic exposure to drugs of abuse [8-10]. The dopamine D3 receptor (D3R) is located in limbic areas, which suggests that D3R may be involved in several processes, such as behavioral sensitization, motivation, emotion, and learning [11-13]. In previous research, it was found that D3R was involved in the reactivity to drug-associated stimuli. The upregulation of D3R happened in animals receiving cocaine or morphine repeatedly in the context that was associated with the cocaine, but not in their home cages $[14,15]$. Selective D3R ligands, such as BP897 and SB-277011A, could reduce conditioned locomotor activity induced by amphetamine and cocaine, respectively $[14,16]$. In addition, Li's study showed that deletion of D3R displayed deficits in the acquisition of morphine-induced behavioral sensitization [17]. The knockout of D3R from the embryo phase, however, may induce a compensatory adaptation, so the results of $\mathrm{D} 3 \mathrm{R}^{-1-}$ mice would accurately clarify the role in the behavioral sensitization. The acute administration of SB-277011A decreased the expression of morphine-induced behavior

\footnotetext{
'Jiangsu Key Laboratory of Neurodegeneration, Department of Pharmacology, Nanjing Medical University, Nanjing 210029, China; ${ }^{2}$ State Key Laboratory of Toxicology and

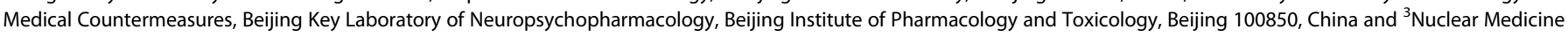
Department, Hainan Branch of the General Hospital of PLA, Sanya 572013, China

Correspondence: Rui Song (songrui1983@vip.163.com) or Jin Li (jinli9802@vip.163.com) or Gang Hu (ghu@njmu.edu.cn)

These authors contributed equally: Yang Lv, Rong-rong $\mathrm{Hu}$
}

Received: 26 March 2018 Accepted: 17 July 2018

Published online: 17 September 2018 
sensitization in the expression phase [15]; however, the clinical translation of SB-277011A has been terminated because of the pharmacokinetic and toxicity problems. In addition, it was reported that the novel dopamine D3 receptor antagonists/partial agonists CAB2-015 and BAK4-54 inhibited oxycodone-taking and oxycodone-seeking behavior in rats self-administration [18]. Whether the effects of CAB2-015 and BAK4-54 on opioid addiction are through D3R in vivo is still unknown. Based on the above literature review, D3R is involved in drug-conditioned responses, and comprehensive research is still needed to investigate the role of D3R in every phase of the morphine-induced morphine sensitization with the highly D3R selective antagonist.

YQA14 is a patented, selective D3 antagonist designed and synthesized by our institute. Our previous studies indicated that YQA14 exhibited a high affinity for D3R with two binding sites, Ki-High $\left(0.68 \times 10^{-4} \mathrm{nM}\right)$ and Ki-Low $(2.11 \mathrm{nM})$, displaying approximately 5,000,000-fold (high affinity) and 150-fold (Low affinity) selectivity over D2R [19]. Furthermore, we have also found that YQA14 does not bind to opioid receptors $(\delta, \mu$, and $k)$ and indicates $>1000$-fold selectivity (unpublished data). Compared with SB-277011A, YQA14 has improved oral bioavailability (>40\%) and a longer half-life $(2 \mathrm{~h})$ in the same human hepatic microsomal enzyme assays [20]. For the pharmacodynamics investigation, YQA14 selectively blocked D3R to attenuate the cocaine-induced self-administration and conditioned place preference (CPP) in WT mice, but not in D3R knockout mice, and YQA14 also reduced the methamphetamine and cocaine induced self-administration and behavior sensitization in rats $[21,22]$. YQA14 can also inhibit the expression and drug-primed reactivation of morphine-induced CPP in rats [23]. These results show that YQA14 has anti-drug addiction effects through specifically binding D3R, and YQA14 is also a highly selective D3R antagonist in vivo, which deserves further study as a candidate in medication development for the treatment of drug abuse and addiction.

The present study used a morphine-induced, context-specific locomotor sensitization model in mice and D3R knockout mice. We first examined the pharmacodynamics of YQA14 in the acquisition and expression following chronic or acute administration. Then, we determined the blocking effects of YQA14 on sensitized locomotion in wild-type and D3R knockout mice.

\section{MATERIALS AND METHODS}

\section{Drugs}

Morphine was obtained from the Beijing Public Security Bureau Forensic Medical Examination Center (China). YQA14 was synthesized by the Beijing Institute of Pharmacology and Toxicology and dissolved in 2-hydroxypropyl- $\beta$-cyclodextrin (Xi'an Deli Biological Chemical Co., Ltd., Xi'an, China), which was used as the vehicle.

\section{Animals}

Male Kunming mice (Beijing Animal Center, Beijing, China) weighing 18-22 $\mathrm{g}$ were used for the investigation of the effects of YQA14. Wild-type (WT) and D3R knockout (D3R ${ }^{-1-}$ ) mice (with a C57BL/6J genetic background, bred at the Beijing Institute Pharmacology and Toxicology) were generated according to previously described methods [24]. All mice were maintained on a 12-h light/dark cycle (lights on at 7:00 AM and lights off at 7:00 PM) with food and water provided ad libitum. All animals were maintained in a facility fully accredited by the Association for Assessment and Accreditation of Laboratory Animal Care International, and all experimental procedures were conducted in accordance with the guidelines established by the Institutional Review Committee for the Use of Animals.

\section{Locomotor sensitization experiment}

The methods for behavioral sensitization in mice were carried out as previously described with minor revisions [25]. Briefly, mice were placed into a locomotor detection chamber ( $40 \mathrm{~L}$ x $40 \mathrm{~W} \times 35$ $\mathrm{H} \mathrm{cm}$, Anilab Instruments) under a video tracking system, and the data were collected automatically and analyzed using the Anilab data analysis system (Anilab Instruments).

To evaluate the effects of YQA14 administration on the morphineinduced behavioral sensitization

The procedure of the morphine-induced behavioral sensitization. Habituation phase (day 1-3): Before administrating drug treatment, all mice were habituated in the test apparatus for three days (30 min per session). Morphine-induced behavioral sensitization and expression phases (day 4-18): Mice in the saline and morphine group were injected with saline $(10 \mathrm{~mL} / \mathrm{kg} \mathrm{sc})$ or morphine $(10 \mathrm{mg} / \mathrm{kg}, \mathrm{sc})$, respectively, and then were placed into the test apparatus immediately, where their locomotion was recorded for $1 \mathrm{~h} /$ day for 7 days (day 4-10). After the test, all mice were returned to their home cages without saline or morphine treatment for a 7-day withdrawal period (day 11-17). In the end, all mice were administered a small dose of morphine $(5 \mathrm{mg} / \mathrm{kg}, \mathrm{sc})$, and locomotion was measured in the detection chambers on day 18.

To investigate the effects of chronic YQA14 treatment on the acquisition of morphine-induced locomotor sensitization (Fig. 1a). The mice in the morphine group were separated into four groups that were administered either vehicle or YQA14 $(6.25,12.5$, or $25 \mathrm{mg} / \mathrm{kg}$, ip) $20 \mathrm{~min}$ before receiving morphine (10 mg/kg, sc). The locomotion of mice was recorded as mentioned above. The mice were not given YQA14 treatment during the withdrawal period or the expression phase.

To examine the effects of acute YQA14 treatment on the expression of morphine-induced locomotor sensitization (Fig. 2a). The mice in the morphine group were separated into four groups that received either vehicle or YQA14 $(6.25,12.5$, or $25 \mathrm{mg} / \mathrm{kg}$, ip) $20 \mathrm{~min}$ before administering morphine $(5 \mathrm{mg} / \mathrm{kg}, \mathrm{sc})$ on the expression day. The activities of the mice were measured for $1 \mathrm{~h}$. The mice were not given YQA14 during the period of acquisition of morphine-induced behavior sensitization or during the withdrawal period.

To investigate the effects of YQA14 on the expression of morphineinduced locomotor sensitization in WT and $D 3 R^{-1-}$ mice (Fig. 3a). To further determine whether the observed pharmacological effects of YQA14 on morphine-induced behavioral sensitization were mediated by blockade of brain DA D3 receptors, we investigated and compared the effects of YQA14 ( $25 \mathrm{mg} / \mathrm{kg}$, ip) on morphine-induced behavioral sensitization in WT and $D 3 \mathrm{R}^{-1-}$ mice.

Habituation phase (day 1-3): Before administering drug treatment, all mice were habituated in the test apparatus for three days (30 min per session). Morphine-induced behavioral sensitization and expression phases (day 4, 7, and 10): All mice were injected with morphine $(10 \mathrm{mg} / \mathrm{kg}, \mathrm{sc})$ and then were placed into the test apparatus immediately for $1 \mathrm{~h}$ per session. After $1 \mathrm{~h}$, all mice were returned to their home cages without morphine treatment for a 7-d withdrawal period (day 11-17). During the withdrawal period, the mice were not given YQA14 treatment. In the end, the mice were separated into two groups (vehicle and YQA14-25 mg/kg) within the $\mathrm{WT}$ and $\mathrm{D} 3 \mathrm{R}^{-1-}$ mice groups that then received either vehicle or YQA14 (25 mg/kg, ip), respectively, $20 \mathrm{~min}$ before administering morphine $(5 \mathrm{mg} / \mathrm{kg}, \mathrm{sc})$ on the expression day (Day 18). The activities of the mice were measured for $1 \mathrm{~h}$. 
a
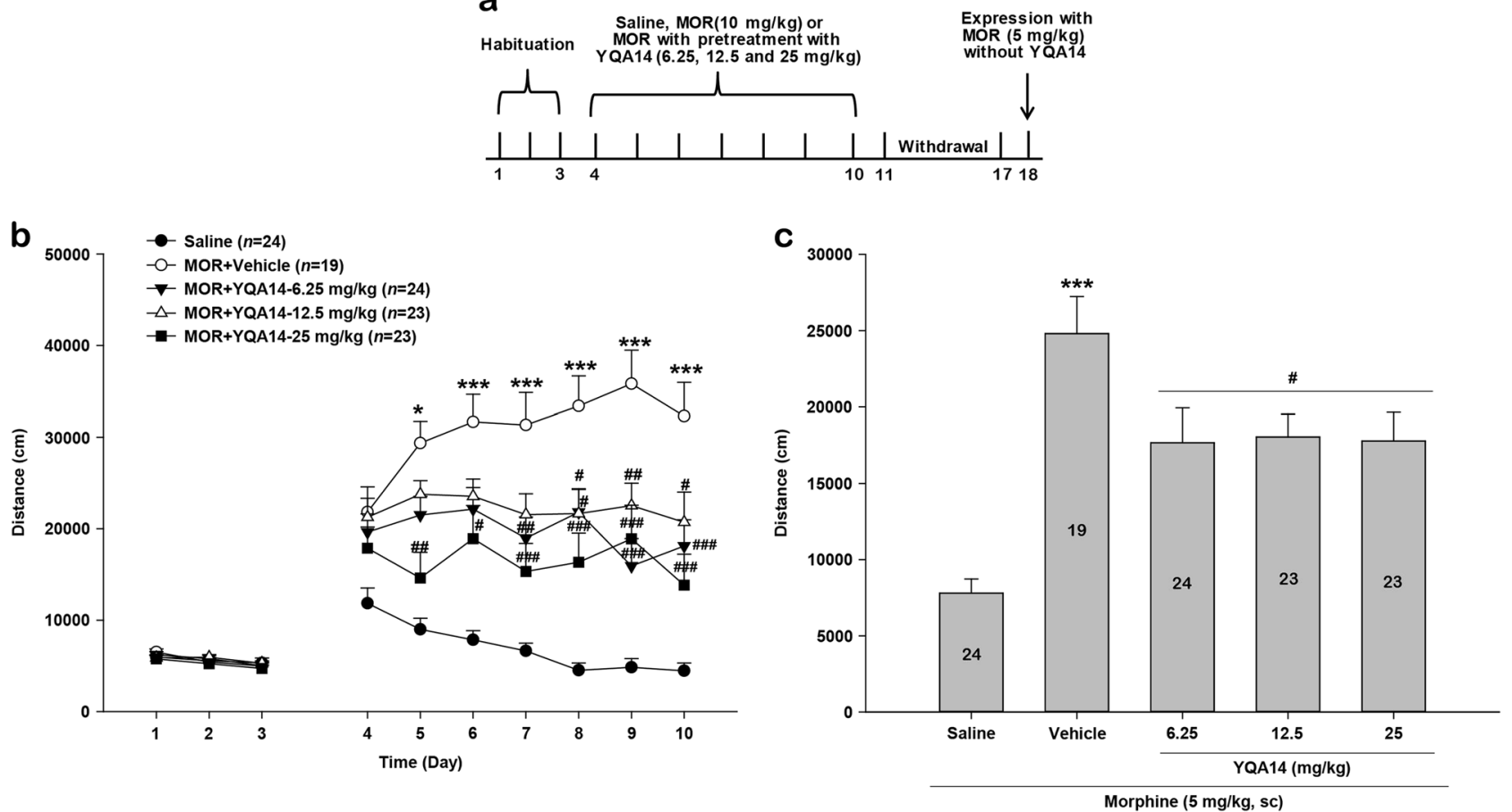

Fig. 1 Effects of the chronic administration of YQA14 on the acquisition of morphine-induced behavioral sensitization in mice. a Experimental protocol for saline, morphine, and YQA14 injections. b Time courses of morphine-induced hyperactivity and behavioral sensitization with or without YQA14 pretreatment. Mean \pm SEM. ${ }^{*} P<0.05$ on Day 5 vs. Day 4; and ${ }^{* * *} P<0.001$ on Day $6,7,8,9$, and 10 vs. Day $4 .{ }^{\#} P<0.05 ;{ }^{\# \#} P<0.01$; and ${ }^{\# \# \#} P<0.001$ vs. the morphine + vehicle group. c Morphine priming produced a significant increase in locomotor activity 7 days after the last morphine injection. ${ }^{* * *} P<0.001$ vs. the saline group. This effect was attenuated in the groups that received chronic treatment with YQA14. ${ }^{\#}<0.05$ vs. morphine treatment alone. Mean \pm SEM

\section{Statistical analysis}

All the data are presented as the mean \pm the standard error of the mean (SEM). Unless specified, the statistical analysis was performed using Sigma Stat. For the acquisition of morphineinduced behavioral sensitization, a two-way analysis of variance (ANOVA) was used in analyzing differences between the YQA14 group and morphine group, within-subjects design was used to assess the effects of morphine-induced locomotor sensitization in mice, and a between-subjects design was used to evaluate the effects of YQA14 on inhibiting morphine-induced locomotor sensitization. For the expression stage, Student $t$-tests were used to compare the differences between two groups (i.e., saline group vs. morphine group), with Bonferroni corrections performed, where necessary, to control for type I errors; the YQA14 groups were analyzed by one-way ANOVA. A two-way ANOVA was also used in analyzing the differences between WT and D3R ${ }^{-1-}$ mice. Individual group comparisons were performed using the Bonferroni method, and differences with $P<0.05$ were considered to be significant.

\section{RESULTS}

Inhibition of the chronic administration of YQA14 on the acquisition and expression of morphine-induced locomotor sensitization

In our previous study, YQA14 $(6.25,12.5$, or $25 \mathrm{mg} / \mathrm{kg})$ treatment did not influence the locomotion on a basal level and could not induce behavioral sensitization [22]. We first compared the effects of repeated morphine $(10 \mathrm{mg} / \mathrm{kg}, \mathrm{sc})$ injection on locomotor behavior with or without YQA14 treatment. Chronic morphine treatment caused significant behavioral sensitization $(P<0.05$ on Day 5 vs. Day 4; and $P<0.001$ on Day $6,7,8,9$, and 10 vs. Day 4 ) in the morphine group mice. Chronic pretreatment of YQA14 with morphine significantly attenuated the acquisition of morphineinduced behavioral sensitization. A two-way ANOVA for repeated measures revealed a significant YQA14 treatment main effect $\left(F_{4,642}=17.78, P<0.001\right)$, time main effect $\left(F_{6,642}=2.10\right.$, $P=0.05)$, and a treatment $\times$ time interaction $\left(F_{24,642}=3.47\right.$, $P<0.001$ ), as shown in Fig. 1b. Post-hoc individual group comparisons revealed a significant difference between the vehicle and YQA14 treatment groups at each dose $(P<0.05, P<0.01$ and $P<0.001$ for $6.25,12.5$, and $25 \mathrm{mg} / \mathrm{kg}$ ).

During the withdrawal period (Days 11-17), all mice were without any treatment. On the expression day, morphine $(5 \mathrm{mg} / \mathrm{kg}, \mathrm{sc})$ administration increased the activity in the vehicle group ( $t=-7.28$, with 41 degrees of freedom, $P<0.001$, compared with the saline group). The YQA14 treatment groups showed significantly decreased expression of morphine-induced locomotor sensitization, as shown in Fig. 1c. A one-way ANOVA revealed a significant main effect of YQA14 treatment $\left(F_{3,84}=\right.$ $3.02, P<0.05)$.

Inhibition of acute pretreatment of YQA14 on the expression of morphine-induced locomotor sensitization

Figure $2 \mathrm{c}$ illustrates that morphine $(5 \mathrm{mg} / \mathrm{kg}, \mathrm{sc})$ administration significantly increased the locomotor response in the vehicle treatment group $(t=-3.92$, with 19 degrees of freedom, $P<$ 0.001 , compared with saline group); however, one single administration pretreatment with YQA14 significantly inhibited this response, as the one-way ANOVA revealed a significant main effect of YQA14 treatment $\left(F_{3,32}=3.54, P<0.05\right)$. Post-hoc individual group comparisons revealed a significant difference between the vehicle and YQA14 treatment groups at a dose of $25 \mathrm{mg} / \mathrm{kg}(t=3.14, P<0.05)$. 


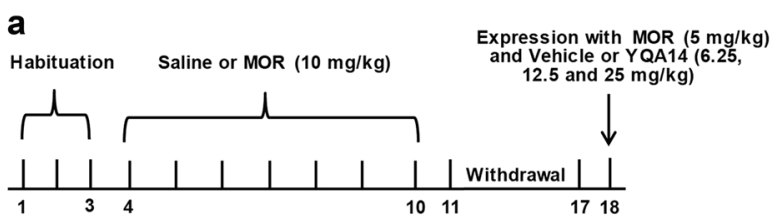

b

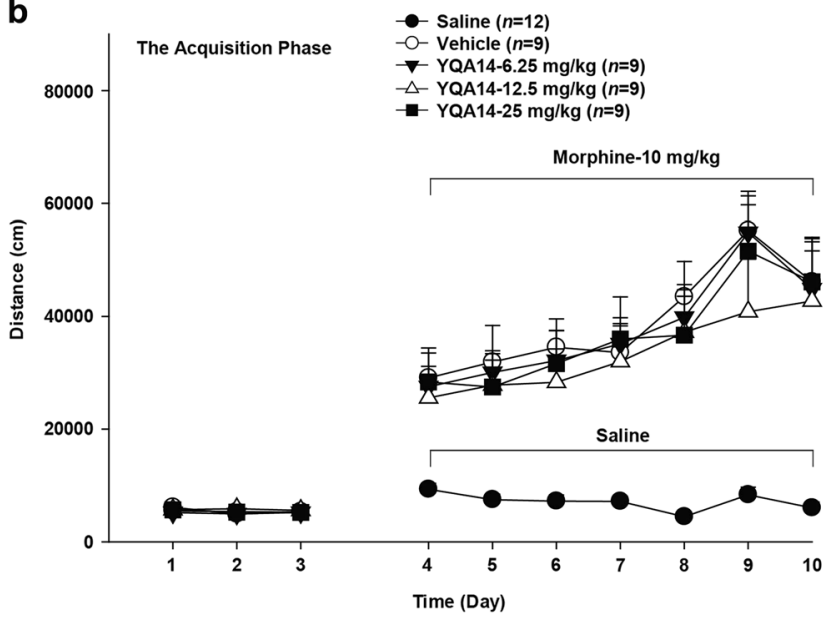

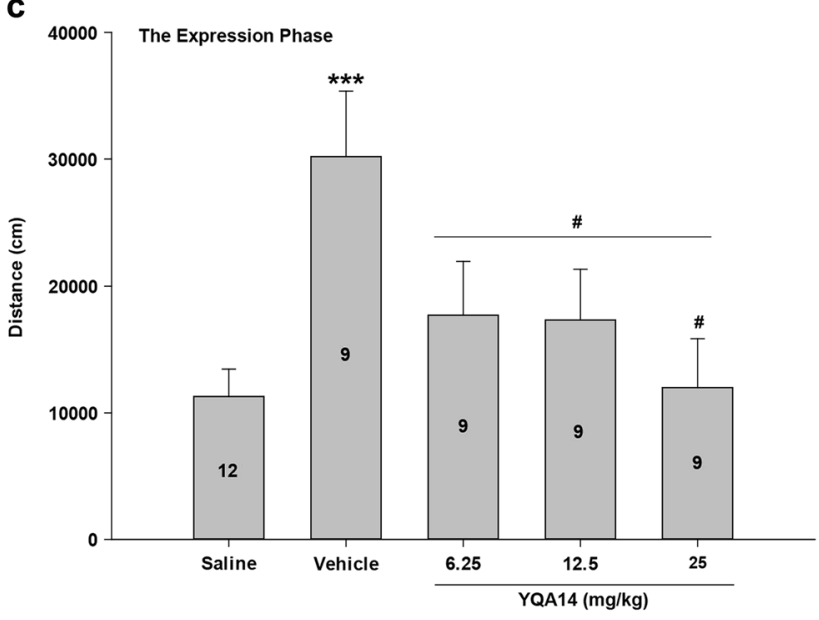

Day 18 - Morphine $(5 \mathrm{mg} / \mathrm{kg}, \mathrm{sc})$

Fig. 2 Effects of the acute administration of YQA14 on the expression of morphine-induced locomotor sensitization in mice. a Experimental protocol for saline, morphine and YQA14 injections. b Time courses of morphine-induced hyperactivity and behavioral sensitization. Mean \pm SEM. c Morphine priming produced a significant increase in locomotor activity 7 days after the last morphine injection. ${ }^{* * * P}<0.001$ vs. the saline group. This effect was attenuated in the groups that received acute pretreatment with YQA 14 . ${ }^{\#} P<0.05$ vs. the morphine treatment group. Mean \pm SEM

a
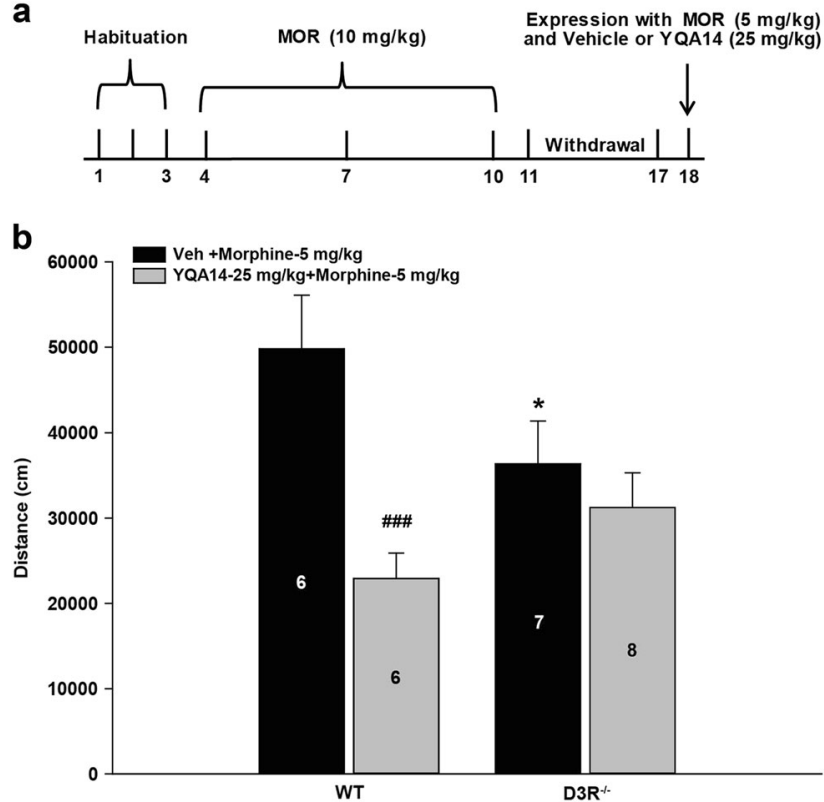

Fig. 3 Effects of the acute administration of YQA14 on the expression of morphine-induced locomotor sensitization in WT and $\mathrm{D}^{2} \mathrm{R}^{-1-}$ mice. a Experimental protocol for morphine and YQA14 injections. b YQA14 administration attenuated the expression of morphine-induced locomotor sensitization in WT mice, but not in $\mathrm{D} 3 \mathrm{R}^{-1-}$ mice. ${ }^{\# \# \#} P<0.001$ vs. morphine treatment in WT mice. ${ }^{*} P<0.05$ compared to the WT morphine group. Mean \pm SEM
Inhibition of acute pretreatment of YQA14 on the expression of morphine-induced locomotor sensitization in WT mice, but not in

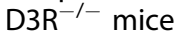

To further determine whether the observed pharmacological effects of YQA14 on morphine-induced behavioral sensitization were mediated by blockade of DA D3 receptors, we compared the effects of YQA14 $(25 \mathrm{mg} / \mathrm{kg}$, ip) pretreatment on morphineinduced behavioral sensitization in WT and $\mathrm{D} \mathrm{R}^{-/-}$mice. In Fig. 3b, a two-way ANOVA for repeated measures revealed a significant YQA14 treatment main effect $\left(F_{1,23}=13.33, P=0.001\right)$, gene main effect $\left(F_{1,23}=0.34, P>0.05\right)$, and a treatment $\times$ gene interaction $\left(F_{1,23}=6.17, P<0.05\right)$. Post hoc individual group comparisons revealed a significant difference between the vehicle and YQA14 treatment groups in the WT mice $(t=4.12, P<0.001)$. In addition, for the vehicle treatments, $\mathrm{D}^{-1-}$ mice showed a significant reduction compared with the WT mice $(t=2.14$, $P<0.05)$; however, there were no significant differences between the vehicle and YQA14 treatment groups in the $\mathrm{D}^{-/-}$mice $(t=0.88, P>0.05)$.

\section{DISCUSSION}

The major findings of this study are as follows: chronic pretreatment of the highly selective D3 receptor antagonist YQA14 with morphine not only inhibited the acquisition of morphine-induced behavioral sensitization but also decreased the expression of morphine-induced behavioral sensitization. Further, one single injection pretreatment with YQA14 attenuated the expression of morphine-induced locomotor sensitization in the expression phase. Furthermore, D3R blockade by YQA14 dose-dependently inhibited the expression of morphine-induced behavioral sensitization in WT mice, but not in $\mathrm{D}^{-1-}$ mice, and the expression of reduction was also displayed in the $\mathrm{D}^{-1-}$ mice. We previously reported that YQA14, itself, neither 
maintained cocaine-induced self-administration in rats nor produced CPP or conditional place aversion (CPA) in mice [21, 22]. Neither acute nor chronic administration of YQA14 influenced basal locomotor behaviors at the series doses of $3.125-25 \mathrm{mg} / \mathrm{kg}$ in the previous studies [22, 26]. Such findings support that YQA14 has neither rewarding nor aversive properties, consistent with the findings with other D3R antagonists, such as SB-277011A and NGB-2904 [27, 28]. Therefore, the highly selective D3R antagonist YQA14 showed the antagonism of morphine-induced behavioral sensitization, which is unlikely due to YQA14-induced aversion or malaise [22].

Behavioral sensitization, described as a progressive and longlasting augmentation of a behavioral response followed by repetitive exposure to the drug administration [29, 30], is one of the most widely used models to investigate drug-induced behavioral plasticity [7, 31-33]. In this model, daily or one single injection pretreatment of YQA14 $(6.25-25 \mathrm{mg} / \mathrm{kg}$, ip) with morphine $(10 \mathrm{mg} / \mathrm{kg}$ in the acquisition phase or $5 \mathrm{mg} / \mathrm{kg}$ in the expression phase) significantly attenuated the acquisition and expression of morphine-induced locomotor sensitization, respectively. The results are also consistent with the other D3R antagonists, such as SB-277011A and nafadotride, to reduce morphine-induced locomotor sensitization [15, 34]. To confirm that the effect of YQA14 on inhibiting locomotor sensitization was due to the blockade of D3R, we also used WT and D3R ${ }^{-1-}$ mice. The acute administration with YQA14 on the expression day inhibited the effects of the expression of morphine-induced

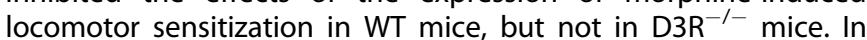
addition, ${\mathrm{D} 3 \mathrm{R}^{-1-}}^{-}$mice also exhibited a significant attenuation followed by vehicle administration after chronic morphine treatment compared with WT mice. This result is similar with the finding by Li et al. [17]. In another study, Compound 16, another high-affinity, D3R-selective antagonist, reduced heroin selfadministration in WT mice, but not in D3R-deficient mice [35]. Together, these findings indicate that the pharmacological blockade of D3R and deletion of the D3R gene show similar reduction effects on morphine-induced locomotor sensitization.

The mechanisms underlying this attenuated morphine-induced behavioral sensitization after D3R deletion or blockade are not fully understood. Accumulating evidence suggests that dopamine is thought to be a key ingredient in both the development of behavioral sensitization to repeated drug administration and the expression of behavioral sensitization upon drug re-administration $[33,36]$. The dopamine projection most often linked to behavioral sensitization is the mesoaccumbens projection from the ventral tegmental area (VTA) to the nucleus accumbens (NAc) [37]. Morphine stimulated $\mu$-opioid receptors in the VTA, which enhanced mesolimbic dopaminergic neurotransmission, especially in the NAc, presumably by the inhibition of GABAergic interneurons, thereby disinhibiting mesolimbic dopaminergic neurons and increasing both somatodendritic and axonal dopamine release $[38-42]$. We noted that $\mathrm{D}^{-/-}$mice displayed a significant increase in basal DA release in the NAc (due to disinhibition after presynaptic D3R loss) and a significant reduction in the NAC DA response to cocaine, which led to attenuated locomotor activity in response to cocaine compared to WT mice and increased cocaine-induced self-administration, which we have interpreted as a compensatory behavioral response to cocaine, secondary to a diminished DA response to cocaine [24]. Similar reduction behavior patterns have been shown for the opioid, where deletion of D3R also displayed the attenuation of locomotor activity in response to acute high morphine $(10 \mathrm{mg} / \mathrm{kg}, \mathrm{sc})$ administration or deficits in the acquisition of locomotor sensitization after chronic morphine administration and increased heroin-induced self-administration [35] (may also be due to reduction in the NAc DA response to opioids), which will need further investigation. Further, the upregulation of $D 3 R$ in the NAC was found in morphine-induced behavioral sensitization through brain-derived neurotrophic factor (BDNF)/ TrkB signaling, and the acute administration of the D3R antagonist SB277011A inhibited the expression of behavioral sensitization and increased the expression of BDNF [15]. In addition, we conducted in vivo microdialysis experiments and found that YQA14 (50 mg/kg, ip) significantly reduced the morphine $(10 \mathrm{mg} /$ $\mathrm{kg}$ )-increased dopamine level in the NAc (data unpublished). Overall, given that D3Rs were deeply involved in morphineinduced behavior sensitization and played an important role in mediating drug-induced reward, a blunted DA response to morphine may underlie the attenuated morphine reward after D3R loss or blockade of YQ14.

In conclusion, the chronic or acute administration of YQA14, a special selective D3R antagonist, induces the persistent inhibition of behavioral sensitization to morphine. In addition, this reduction is due to the total block of dopamine D3R in mice. The data provide additional support for the potential utility of the selective D3 receptor antagonist YQA14 in the treatment of opioid addiction.

\section{ACKNOWLEDGEMENTS}

This work was supported by the National Key Research and Development Program of China (2016YFC0800907), Medical Innovation Program (16CXZ033), National Key Basic Research Program (2015CB553504), Natural Science Foundation of China (No. 81573405) and Beijing Nova Program (xx2014A014).

\section{AUTHOR CONTRIBUTIONS}

$\mathrm{YL}, \mathrm{RRH}$ and $\mathrm{MJ}$ conducted the experiments and analyzed the data. RS designed the experiments. $\mathrm{YL}$ wrote the draft manuscript. TZ bred the mice. NW initiated this project. RS, JL and GH supervised the experiments and revised the manuscript. We thank Ri-fang Yang (Beijing Institute of Pharmacology and Toxicology) for providing YQA14.

\section{ADDITIONAL INFORMATION}

Competing interests: The authors declare no competing interests.

\section{REFERENCES}

1. The Annual Report of Drug Abuse Monitoring in China [China food and drug administration 2017 Aug 11]. http://www.sda.gov.cn/WS01/CL0844/175994.html.

2. Potter JS, Marino EN, Hillhouse MP, Nielsen S, Wiest K, Canamar CP, et al. Buprenorphine/naloxone and methadone maintenance treatment outcomes for opioid analgesic, heroin, and combined users: findings from starting treatment with agonist replacement therapies (START). J Stud Alcohol Drugs. 2013;74:605-13.

3. Fiellin DA, Schottenfeld RS, Cutter CJ, Moore BA, Barry DT, O'Connor PG, et al. Primary care-based buprenorphine taper vs. maintenance therapy for prescription opioid dependence: a randomized clinical trial. JAMA Intern Med. 2014;174:1947-54.

4. Novick DM, Salsitz EA, Joseph $H$, Kreek MJ. Methadone medical maintenance: an early 21st-century perspective. J Addict Dis. 2015;34:226-37.

5. Koob GF, Le Moal M. Drug addiction, dysregulation of reward, and allostasis. Neuropsychopharmacology. 2001;24:97-129.

6. Robinson TE, Berridge KC. Addiction. Annu Rev Psychol. 2003:54:25-53.

7. Robinson TE, Browman KE, Crombag HS, Badiani A. Modulation of the induction or expression of psychostimulant sensitization by the circumstances surrounding drug administration. Neurosci Biobehav Rev. 1998;22:347-54.

8. Hyman SE, Malenka RC. Addiction and the brain: the neurobiology of compulsion and its persistence. Nat Rev Neurosci. 2001;2:695-703.

9. Kalivas PW, O'Brien C. Drug addiction as a pathology of staged neuroplasticity. Neuropsychopharmacology. 2008;33:166-80.

10. Luscher C, Malenka RC. Drug-evoked synaptic plasticity in addiction: from molecular changes to circuit remodeling. Neuron. 2011;69:650-63.

11. Bouthenet ML, Souil E, Martres MP, Sokoloff $P$, Giros B, Schwartz JC. Localization of dopamine D3 receptor mRNA in the rat brain using in situ hybridization histochemistry: comparison with dopamine D2 receptor mRNA. Brain Res. 1991;564:203-19.

12. Gurevich EV, Joyce JN. Distribution of dopamine D3 receptor expressing neurons in the human forebrain: comparison with D2 receptor expressing neurons. Neuropsychopharmacology. 1999;20:60-80. 
13. Khaled MA, Pushparaj A, Di Ciano P, Diaz J, Le Foll B. Dopamine D3 receptors in the basolateral amygdala and the lateral habenula modulate cue-induced reinstatement of nicotine seeking. Neuropsychopharmacology. 2014;39:3049-58.

14. Le Foll B, Frances H, Diaz J, Schwartz JC, Sokoloff P. Role of the dopamine D3 receptor in reactivity to cocaine-associated cues in mice. Eur J Neurosci. 2002;15:2016-26.

15. Liang J, Zheng X, Chen J, Li Y, Xing X, Bai Y, et al. Roles of BDNF, dopamine $D(3)$ receptors, and their interactions in the expression of morphineinduced context-specific locomotor sensitization. Eur Neuropsychopharmacol. 2011;21:825-34.

16. Aujla $H$, Sokoloff $P$, Beninger RJ. A dopamine D3 receptor partial agonist blocks the expression of conditioned activity. Neuroreport. 2002;13:173-6.

17. Li T, Hou Y, Yan CX, Chen T, Zhao Y, Li SB. Dopamine D3 receptor knock-out mice display deficits in locomotor sensitization after chronic morphine administration. Neurosci Lett. 2010;485:256-60.

18. You ZB, Gao JT, Bi GH, He Y, Boateng C, Cao J, et al. The novel dopamine D3 receptor antagonists/partial agonists CAB2-015 and BAK4-54 inhibit oxycodone-taking and oxycodone-seeking behavior in rats. Neuropharmacology. 2017;126:190-9.

19. Song R, Yang RF, Wu N, Su RB, Li J, Peng XQ, et al. YQA14: a novel dopamine D-3 receptor antagonist that inhibits cocaine self-administration in rats and mice, but not in D-3 receptor-knockout mice. Addict Biol. 2012;17:259-273.

20. Liu F, Zhuang X, Yang C, Li Z, Xiong S, Zhang Z, et al. Characterization of preclinical in vitro and in vivo ADME properties and prediction of human PK using a physiologically based pharmacokinetic model for YQA-14, a new dopamine D3 receptor antagonist candidate for treatment of drug addiction. Biopharm Drug Dispos. 2014;35:296-307.

21. Song R, Yang RF, Wu N, Su RB, Li J, Peng XQ, et al. YQA14: a novel dopamine D3 receptor antagonist that inhibits cocaine self-administration in rats and mice, but not in D3 receptor-knockout mice. Addict Biol. 2012;17:259-73.

22. Song R, Zhang HY, Peng XQ, Su RB, Yang RF, Li J, et al. Dopamine D3 receptor deletion or blockade attenuates cocaine-induced conditioned place preference in mice. Neuropharmacology. 2013;72:82-7.

23. Hu R, Song R, Yang R, Su R, Li J. The dopamine $D(3)$ receptor antagonist YQA14 that inhibits the expression and drug-prime reactivation of morphineinduced conditioned place preference in rats. Eur J Pharmacol. 2013;720: 212-7.

24. Song $\mathrm{R}$, Zhang HY, Li X, Bi GH, Gardner EL, Xi ZX. Increased vulnerability to cocaine in mice lacking dopamine D3 receptors. Proc Natl Acad Sci U S A. 2012;109:17675-80.

25. Sun L, Song R, Chen Y, Yang RF, Wu N, Su RB, et al. A selective D3 receptor antagonist YQA14 attenuates methamphetamine-induced behavioral sensitization and conditioned place preference in mice. Acta Pharmacol Sin. 2016;37: 157-65.

26. Song R, Bi GH, Zhang HY, Yang RF, Gardner EL, Li J, et al. Blockade of D3 receptors by YQA14 inhibits cocaine's rewarding effects and relapse to drug-seeking behavior in rats. Neuropharmacology. 2014;77:398-405.
27. Vorel SR, Ashby CR Jr, Paul M, Liu X, Hayes R, Hagan JJ, et al. Dopamine D3 receptor antagonism inhibits cocaine-seeking and cocaine-enhanced brain reward in rats. J Neurosci. 2002;22:9595-9603.

28. Xi ZX, Newman AH, Gilbert JG, Pak AC, Peng XQ, Ashby CR Jr, et al. The novel dopamine D3 receptor antagonist NGB 2904 inhibits cocaine's rewarding effects and cocaine-induced reinstatement of drug-seeking behavior in rats. Neuropsychopharmacology. 2006;31:1393-1405.

29. Robinson TE, Becker JB. Enduring changes in brain and behavior produced by chronic amphetamine administration: a review and evaluation of animal models of amphetamine psychosis. Brain Res. 1986;396:157-98.

30. Segal DS, Mandell AJ. Long-term administration of $d$-amphetamine: progressive augmentation of motor activity and stereotypy. Pharmacol Biochem Behav. 1974;2:249-55.

31. Steketee JD, Kalivas PW. Drug wanting: behavioral sensitization and relapse to drug-seeking behavior. Pharmacol Rev. 2011;63:348-65.

32. Anagnostaras SG, Robinson TE. Sensitization to the psychomotor stimulant effects of amphetamine: modulation by associative learning. Behav Neurosci. 1996;110:1397-1414.

33. Pierce RC, Kalivas PW. A circuitry model of the expression of behavioral sensitization to amphetamine-like psychostimulants. Brain Res Brain Res Rev. 1997;25:192-216.

34. Liu XS, Hou Y, Yan TL, Guo YY, Han W, Guan FL, et al. Dopamine D3 receptorregulated $\mathrm{NR} 2 \mathrm{~B}$ subunits of $\mathrm{N}$-methyl-d-aspartate receptors in the nucleus accumbens involves in morphine-induced locomotor activity. CNS Neurosci Ther. 2014;20:823-9.

35. Boateng CA, Bakare OM, Zhan J, Banala AK, Burzynski C, Pommier E, et al. High affinity dopamine D3 receptor (D3R)-selective antagonists attenuate heroin self-administration in wild-type but not D3R knockout mice. J Med Chem. 2015;58:6195-6213.

36. Robinson $T E$, Berridge $K C$. The neural basis of drug craving: an incentivesensitization theory of addiction. Brain Res Brain Res Rev. 1993;18:247-291.

37. Le Moal $\mathrm{M}$, Simon $\mathrm{H}$. Mesocorticolimbic dopaminergic network: functional and regulatory roles. Physiol Rev. 1991;71:155-234.

38. Kalivas PW, Duffy P. Effect of acute and daily cocaine treatment on extracellular dopamine in the nucleus accumbens. Synapse. 1990;5:48-58.

39. Spanagel R, Herz A, Shippenberg TS. Opposing tonically active endogenous opioid systems modulate the mesolimbic dopaminergic pathway. Proc Natl Acad Sci U S A. 1992;89:2046-2050.

40. Johnson SW, North RA. Opioids excite dopamine neurons by hyperpolarization of local interneurons. J Neurosci. 1992;12:483-488.

41. Klitenick MA, DeWitte $P$, Kalivas PW. Regulation of somatodendritic dopamine release in the ventral tegmental area by opioids and GABA: an in vivo microdialysis study. J Neurosci. 1992;12:2623-2632.

42. Devine $D P$, Leone $P$, Pocock $D$, Wise RA. Differential involvement of ventral tegmental mu, delta and kappa opioid receptors in modulation of basal mesolimbic dopamine release: in vivo microdialysis studies. J Pharmacol Exp Ther. 1993;266:1236-1246. 